\title{
Three-Frequency Resonances in Coupled Phase-Locked Loops
}

\author{
Oscar Calvo, Julyan H. E. Cartwright, Diego L. González, \\ Oreste Piro, \& Francesco Sportolari
}

\begin{abstract}
We construct an experimental model of a nonlinear dynamical system with three frequencies. With this analog electronic circuit made up of quasiperiodically forced coupled phase-locked loops, we investigate the structure of the scaling of three-frequency resonances or lockings in the dynamics. We hypothesize and confirm experimentally that for weak coupling the three-frequency resonance with the largest frequency-locked plateau in the space of parameters in the interval between two adjacent resonances $p / q$ and $r / s$ is given by the mediant $(p+r) /(q+s)$. We expect this to be universal behavior in systems of three coupled oscillators.
\end{abstract}

\section{INTRODUCTION}

$\mathrm{T}$ HE last decades of this millennium have seen a revolution in our understanding of complex and nonlinear systems driven by the application of the mathematics of dynamical systems theory to diverse fields of science, ranging from physics, through engineering, to chemistry and biology.

Many of these breakthroughs have been possible thanks to insight into the behavior of discrete and continuous systems gained through intensive numerical calculations. The bulk of these calculations have been performed using digital circuits. However, there is a growing interest in analog electronic circuits as systems where theoretical predictions can be tested in a more realistic environment that that of numerical model simulation, as experimental systems where new interesting dynamical properties can be discovered, and as powerful emulators of real complex systems in practical applications [1], [2], [3], [4], [5], [6], [7].

A particularly important aspect of the new viewpoint that we have arrived at in the study of dynamical systems is that of universality. There are many facets of the behavior of dynamical systems that are universal across classes of systems in very different fields of science. Within a class, we can predict the qualitative and in some cases quantitative behavior of the system without having investigated the details of each case.

The dynamics of nonlinear systems with two interacting frequencies has been thoroughly investigated in many theoretical and experimental studies, including many studies of electronic circuits consisting of forced and coupled nonlinear oscillators. It is now well understood how the resonances or lockings found in these systems arise, and how they are distributed in the parameter space [8], [9], [10], [11], [12]. Only recently, how-

\footnotetext{
Oscar Calvo is with L.E.I.C.I. at the Universidad Nacional de La Plata, Argentina. Email: calvo@athos.fisica.unlp.edu.ar.

Julyan Cartwright is with the Instituto Andaluz de Ciencias de la Tierra, Granada, Spain. Email: julyan@galiota.uib.es, Web http://formentor.uib.es/ julyan.

Diego González and Francesco Sportolari are with the Istituto Lamel, CNR, Bologna, Italy. Email: gonzalez@lamel.bo.cnrit and sportolari@lamel.bo.cnr.it.

Oreste Piro is with IMEDEA on the campus of the Universitat de les Illes Balears, Spain. Email: piro@imedea.uib.es, Web http://www.imedea.uib.es/ piro.
}

ever, has attention been brought to bear on the more complex problem of three interacting frequencies. It is known that threefrequency systems also have a structure of resonances, but here there are three-frequency resonances as well as the type found in systems with only two frequencies. Previous investigation of three-frequency systems has shown that there is a web of resonances throughout the parameter space [13]. Experiments with electronic circuits [14], [15], [16] have confirmed theoretical results, and provided much data for theorists. The broad picture of three-frequency systems is clear from these studies, but the question of scaling laws for the relative sizes of resonances in small intervals of parameter space has not been addressed.

In this paper we investigate universal aspects of the fine structure of the nonlinear dynamics of a three-frequency system with weak coupling. We develop a theoretical prediction about the distribution and hierarchy of three-frequency resonances in the parameter space. We then construct an analog circuit of quasiperiodically forced coupled phase-locked loops. We focus our attention on the three-frequency resonances in the output of the circuit, and use it to test our scaling hypothesis.

The paper is organized as follows: in Section II we give a succinct review of the theoretical basis for the scaling of resonances in two-frequency systems, and we build on that to propose a hypothesis for the scaling of three-frequency resonances in three-frequency systems. In Section III we describe the analog circuit of coupled phase-locked loops we use, in Section IV we analyse the behavior of the circuit and test the validity of our scaling hypothesis; we end in Section V with conclusions.

\section{THREE-FREQUENCY RESONANCES}

Nonlinear systems with competing frequencies show the phenomenon of resonance, also termed phase-, mode- or frequency locking, in which the system locks into a resonant periodic response which has a rational frequency ratio. Resonance was first observed and explained in 1665 by Huygens [17], [18], [19] in two pendulum clocks coupled by a common mounting. The amount of resonance increases with coupling strength, from none in the uncoupled linear regime, to a critical situation where the system is locked into resonance at all values of the frequency ratio. The subcritical system has quasiperiodic responses between different lockings, while at supercritical values of the coupling strength, chaotic as well as periodic and quasiperiodic responses may occur. Resonance phenomena were first noted in the context of electronics by van der Pol [20], [21]. In the intervening seventy years, resonances have been investigated theoretically and experimentally in many nonlinear systems, and their distribution in parameter space is now well understood, from the number theoretical concept of Farey trees [8], [9], [10], [11], 
[12]. However, all this applies to resonances generated by the interaction of two frequencies. Far less is known, by comparison, when there are three or more interacting frequencies.

Adding another frequency allows new phenomena to take place. Now as well as the possibility of all three frequencies having a rational relation, to form a (two-frequency) resonance as before, there is a further possibility: that of a three-frequency resonance, also known as a weak resonance or partial mode locking. Three-frequency resonances are given by the nontrivial solutions of the equation $a f_{0}+b f_{1}+c f_{2}=0$, where $a, b$, and $c$ are integers, $f_{1}$ and $f_{2}$ are the forcing frequencies, and $f_{0}$ is the resonant response. Three-frequency resonances form a web in the parameter space of the frequencies [13], [14], [15], [16]. In this section we review the novel number-theoretical results [22], [23] leading to the present investigations. We should like to discover the local scaling laws governing the relative sizes of neighboring three-frequency resonances. To do this we must have recourse to some number theory of rational approximations. Firstly, we revise the situation for the case of two frequencies, then we extend this to systems in which there are three interacting frequencies.

\section{A. Continued fraction approximations for two frequencies}

Consider a two-frequency system with autonomous frequency $f_{0}$ and external frequency $f_{1}$. Let $\tilde{f}=f_{1} / f_{0}$. Our aim is to define a sequence of rationals that converges to $\tilde{f}$. Strong convergence is measured with the metric (Kinchin's metric of the second kind [24])

$$
\left\|\tilde{f}-\frac{p_{i}}{q_{i}}\right\|=\left|q_{i} \tilde{f}-p_{i}\right| .
$$

$p_{n} / q_{n}$ is a best rational approximation if

$$
\left\|\tilde{f}-\frac{p_{n}}{q_{n}}\right\|<\left\|\tilde{f}-\frac{p_{i}}{q_{i}}\right\|
$$

for all $\left(p_{i}, q_{i}\right)$ for any $q_{i} \leq q_{n}$. Given $\tilde{f}, p_{n}$ and $q_{n}$ are produced by expanding $\tilde{f}$ in continued fractions $\tilde{f}=\left(a_{1}, a_{2}, a_{3}, \ldots\right)$, and truncating the expansion as $p_{n} / q_{n}=\left(a_{1}, a_{2}, a_{3}, \ldots a_{n}\right)$ [25]. The $p_{n} / q_{n}$ are then the strong convergents of $\tilde{f}$. They give the sequence of fractions with lowest monotonically increasing denominators that converges to $\tilde{f}$.

\section{B. The Farey tree for two frequencies}

The physically reasonable hypothesis invoked to explain the local ordering of the hierarchy of (two-frequency) resonances is that the smaller the denominator, the larger the width of the resonance in parameter space. The fraction with smallest denominator between $p / q$ and $r / s$, if they are sufficiently close so that $|q r-p s|=1$, when they are called adjacents, is $(p+r) /(q+s)$. This fraction, known as the mediant, then gives the most important resonance in the interval between the resonances $p / q$ and $r / s$. Repeatedly performing the mediant operation

$$
\frac{p}{q} \oplus \frac{r}{s}=\frac{p+r}{q+s}
$$

on a pair of adjacent rationals, we obtain a Farey tree. For weak coupling, the Farey tree provides a qualitative local ordering of two-frequency resonances [8], [9], [10], [11], [12].

\section{Continued fraction approximations for three frequencies}

Now consider the case of three frequencies, one internal $f_{0}$, and two external $f_{1}$ and $f_{2}$. We may divide through by the autonomous frequency $f_{0}$, to give $f_{1}^{\dagger}=f_{1} / f_{0}$, and $f_{2}^{\dagger}=f_{2} / f_{0}$. We now aim to come up with two convergent sequences of rationals with the same denominator, $p_{n} / k_{n}$ and $q_{n} / k_{n}$, which are strong convergents to $f_{1}^{\dagger}$ and $f_{2}^{\dagger}$ respectively.

As before, strong convergence is measured through the metric

$$
\left\|\left(f_{1}^{\dagger}, f_{2}^{\dagger}\right)-\left(\frac{p_{i}}{k_{i}}, \frac{q_{i}}{k_{i}}\right)\right\|=\left|k_{i}\left(f_{1}^{\dagger}, f_{2}^{\dagger}\right)-\left(p_{i}, q_{i}\right)\right| .
$$

Thus $\left(p_{n} / k_{n}, q_{n} / k_{n}\right)$ are best rational approximants if

$$
\left\|\left(f_{1}^{\dagger}, f_{2}^{\dagger}\right)-\left(\frac{p_{n}}{k_{n}}, \frac{q_{n}}{k_{n}}\right)\right\|<\left\|\left(f_{1}^{\dagger}, f_{2}^{\dagger}\right)-\left(\frac{p_{i}}{k_{i}}, \frac{q_{i}}{k_{i}}\right)\right\|
$$

for all triplets of integers $\left(p_{i}, q_{i}, k_{i}\right)$ for any $k_{i} \leq k_{n}$.

So we may write

$$
\begin{aligned}
& \varepsilon_{1}=\left\|\frac{p_{n}}{k_{n}}-f_{1}^{\dagger}\right\|=\left|k_{n} f_{1}^{\dagger}-p_{n}\right|, \\
& \varepsilon_{2}=\left\|\frac{q_{n}}{k_{n}}-f_{2}^{\dagger}\right\|=\left|k_{n} f_{2}^{\dagger}-q_{n}\right|,
\end{aligned}
$$

where we wish to obtain the integers $p_{n}, q_{n}$ and $k_{n}$. This general problem has not been solved [26], [27], however, we may set $\varepsilon_{1}=\varepsilon_{2}$, so that both approximations should be equally good or bad. Taking $\varepsilon_{1}=\varepsilon_{2}$ is an ansatz to simplify the problem that may, or may not, prove correct; as we shall see later, it leads to results that are confirmed both in numerical simulations and experimentally. Note that the work cited above of Kim \& Ostlund [26], [27], is dedicated to the organization of two-frequency resonances in three-frequency systems, whereas here we are concerned with the more general three-frequency resonances.

If we then set $\varepsilon_{1}=\varepsilon_{2}$, we can equate

$$
\left|k_{n} f_{1}^{\dagger}-p_{n}\right|=\left|k_{n} f_{2}^{\dagger}-q_{n}\right|
$$

and ask what is $k_{n}$. There are two solutions

$$
k_{n}=\frac{q_{n} \pm p_{n}}{f_{2}^{\dagger} \pm f_{1}^{\dagger}} .
$$

At this point we must remember that $k_{n}$ is an integer, so these solutions require that the frequencies be rescaled by $f_{2}^{\dagger} \pm f_{1}^{\dagger}$. For which we define for the first solution

$$
\tilde{f}_{1}=\frac{f_{1}^{\dagger}}{f_{1}^{\dagger}+f_{2}^{\dagger}}, \quad \tilde{f}_{2}=\frac{f_{2}^{\dagger}}{f_{1}^{\dagger}+f_{2}^{\dagger}},
$$

and similarly for the other solution

$$
\tilde{f}_{1}^{*}=\frac{f_{1}^{\dagger}}{f_{2}^{\dagger}-f_{1}^{\dagger}}, \quad \tilde{f}_{2}^{*}=\frac{f_{2}^{\dagger}}{f_{2}^{\dagger}-f_{1}^{\dagger}} .
$$

The two solutions give rise to different $\varepsilon$ 's

$$
\begin{aligned}
\varepsilon & =\left|\left(p_{n}+q_{n}\right) \tilde{f}_{1}-p_{n}\right|=\left|\left(p_{n}+q_{n}\right) \tilde{f}_{2}-q_{n}\right| \\
\varepsilon^{*} & =\left|\left(q_{n}-p_{n}\right) \tilde{f}_{1}^{*}-p_{n}\right|=\left|\left(q_{n}-p_{n}\right) \tilde{f}_{2}^{*}-q_{n}\right|
\end{aligned}
$$


Coupled phase-locked loop circuits are also of interest to industry. For example, two coupled phase-locked loops are used to synchronize geographically separated timing clocks [30]. These kinds of systems have also been studied as dynamical systems in order to determine the boundaries of regions of safe operation, that is, regions free of irregular or chaotic behavior [2], [7].

Here our circuit consists of two coupled voltage-controlled oscillators forced with two independent external forces of frequencies $f_{1}$ and $f_{2}$. In fig 3 we show the electrical scheme of the circuit implementation. The outputs of the two phaselocked loops are sent to a type 1 phase comparator. The type 1 phase comparator of CD 4046A is an exclusive OR network that exhibits a triangular shaped response after low-pass filtering. The error signal is fed back to both voltage-controlled oscillators, and passes through an overall adjustable amplifier to provide control over the coupling strength; we are interested in the weak coupling regime in this work. Inverted and direct versions of the error signal are sent to oscillators one and two respectively; this inversion of the error signal in one of the paths is necessary for the stability of the circuit. Feedback signals enter the voltage-controlled-oscillator control pins through appropriate adder circuits. The adders also allow independent coupling with the external forces and tuning of the internal frequencies through application of adjustable DC levels.

In a phase-locked loop one normally uses the voltagecontrolled oscillator and one of the two phase comparators from the integrated circuit. In our experiment we use a voltagecontrolled oscillator for each phase-locked loop and a type 1 phase comparator of one of the two CD 4046As. The rest of the circuit consists of external linear adders and amplifiers. The low-pass filter is also external as in normal phase-locked-loop circuits.

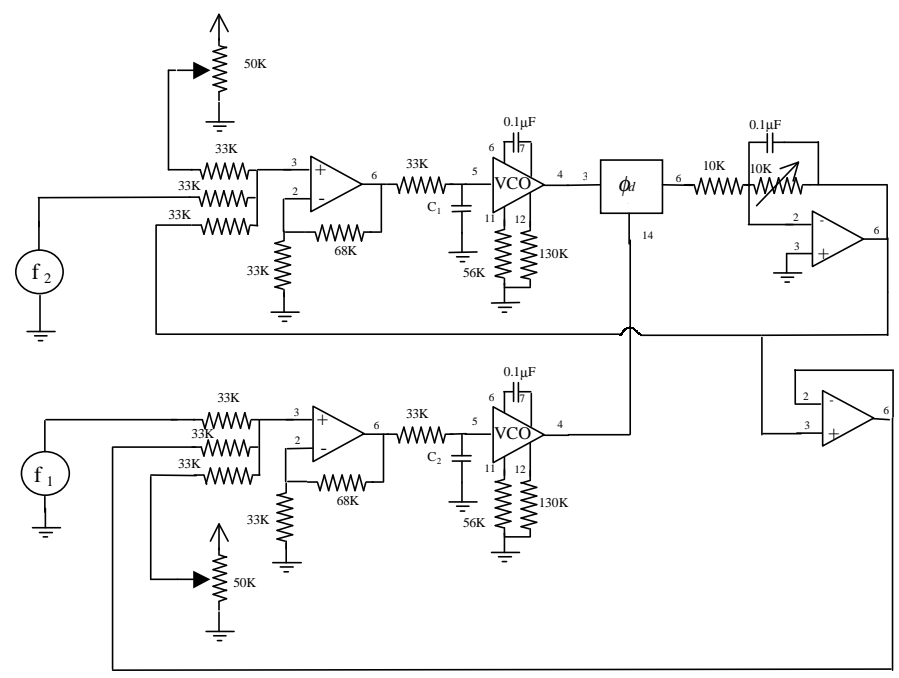

Fig. 3. The circuit. The output of the voltage controlled oscillator (VCO) sections of both digital phase-locked loop devices are sent to an exclusive OR port for phase comparison. The phase comparator output is returned, after low-pass filtering, to both VCO inputs. An amplifier in the feedback path controls the coupling strength between the oscillators. Appropriate operational adders on both VCO inputs allow the external forcing of the oscillators. Stable phase-locked responses between oscillators also require an additional unit gain inverter prior to one of the device inputs.

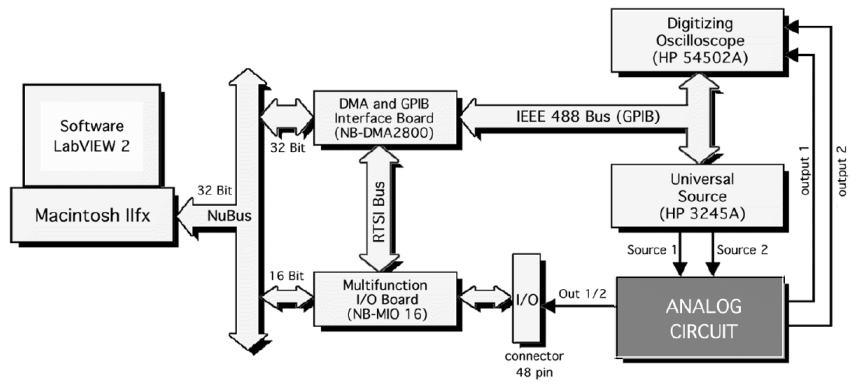

Fig. 4. The experimental setup. Apart from the circuit itself, we use an HP 3425A function generator, a digitizer, and a Macintosh running the graphical control software LabView 2 for overall control of the experiment.

\section{EXPERIMENTAL SETUP AND RESUlTS}

Figure 4 shows a block diagram of the experimental setup. The external forces are generated by an HP 3245A two-channel digital function generator. As a control parameter we use the DC offset of one of the external forces; a change in the mean value of an external force gives a linear change in the natural frequency of the associated oscillator: we vary the DC offset of $f_{1}$ in steps of $1 \mathrm{mV}$. The outputs of the oscillators are digitized at a rate of $40 \mathrm{kHz}$, and saved for subsequent analysis. Graphical control software (a Macintosh running LabView 2) is used to control the overall experiment. A controllable time from the start of any set of data can be chosen in order to allow transients to die out. After this time the fundamental frequency is calculated by a virtual instrument frequency meter.

The circuit shows 1/1 synchronization between the two oscillators for a wide range of excitation and system parameters. Typically, the fundamental frequency of both outputs remains constant in a finite interval of DC offset. The frequency value of the fundamental determines, together with the frequency values of the two external forces, a three-frequency resonance. Experimental data from the circuit are shown in fig. 5 together with the predicted hierarchy of resonances described by our mediant hypothesis of Section II. The two external frequencies are here fixed at $f_{1}=2100 \mathrm{~Hz}$ and $f_{2}=3600 \mathrm{~Hz}$ respectively. These values correspond to the example we gave in Section IID.

In fig. 5 the first level is defined by the two adjacents $f_{2} / 7 \approx$ $514.3 \mathrm{~Hz}$ and $f_{1} / 4=525 \mathrm{~Hz}$, which are subharmonics of the external frequencies. The mediant $\tilde{f}_{s}$ between these two solutions corresponds to $\left(f_{1}+f_{2}\right) /(4+7) \approx 518.2 \mathrm{~Hz}$. Clearly, this is the largest stability region in this parameter interval. Subsequent levels in the hierarchy confirm the mediant hypothesis up to the limit of resolution of the graphic. In fig. 6 we show a power spectrum for one of the outputs in the parameter region of $\tilde{f}_{s}$. The spectrum is dominated by the $\tilde{f}_{s}$ peak, which is at least $10 \mathrm{~dB}$ greater than the components at the external frequencies. This shows that the subharmonic frequency mainly determines the dynamics of the system. Moreover, we can see that the spectrum is very complicated in the low frequency region, owing principally to the presence of several minor peaks. The principal spacing between peaks is $\varepsilon \tilde{f}_{s} \approx 27.29 \mathrm{~Hz}$, which corresponds to beats between the external forces and appropriate harmonics of $\tilde{f}_{s}$ (see fig. 1 ). 


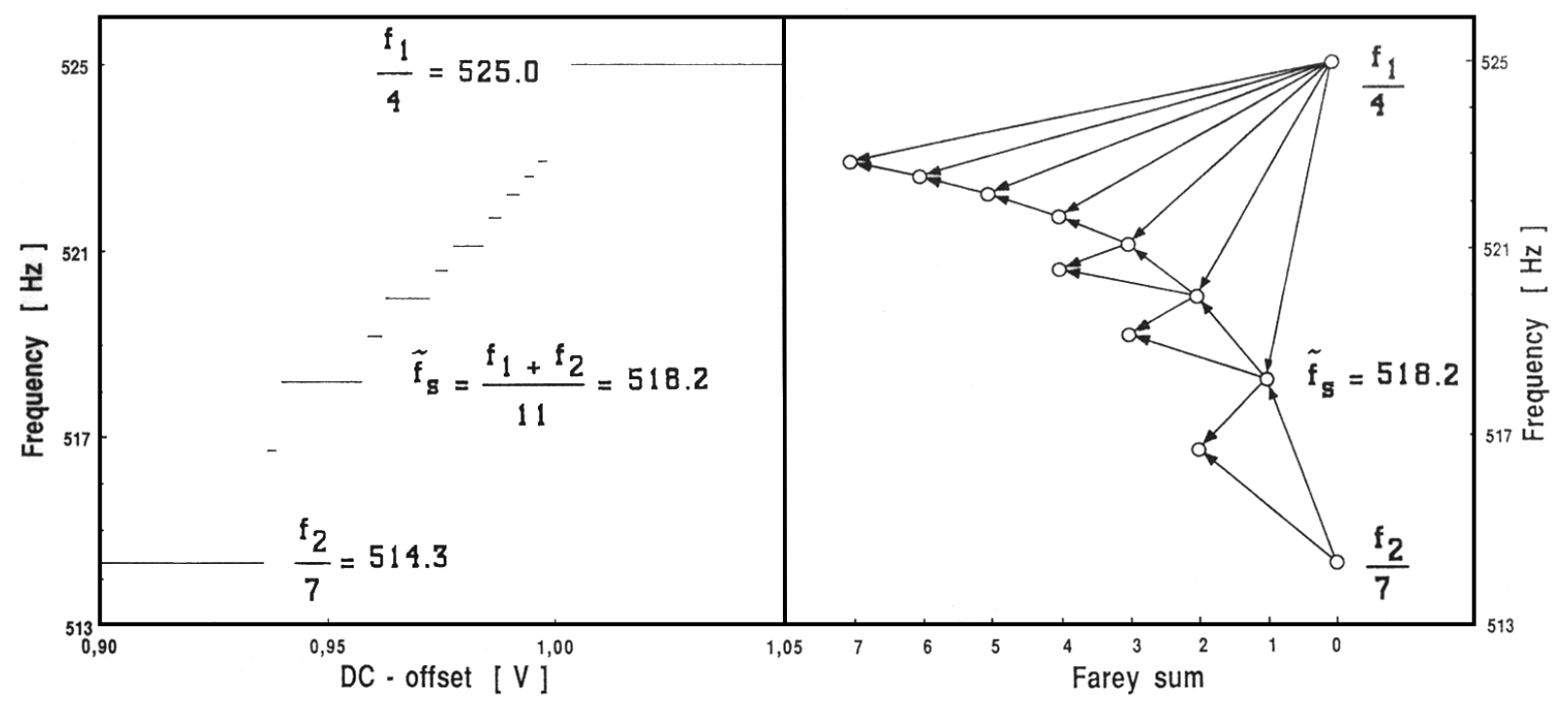

Fig. 5. Experimental results - a three-frequency Devil's staircase. The external frequencies $f_{1}$ and $f_{2}$ are here fixed at $2100 \mathrm{~Hz}$ and $3600 \mathrm{~Hz}$. On the left is plotted the third frequency of a three-frequency resonance against a control parameter (the DC offset of one of the external forces) for all resonances with plateaux larger than a certain size. On the right we see the hierarchy of three-frequency resonances predicted by the mediant hypothesis starting from the parents $f_{1} / 4$ and $f_{2} / 7$. At each level in the hierarchy, the daughter resonance formed by the mediant between two adjacent parents is seen to be the largest in its interval.

As $4 / 7$ is a convergent of $f_{1} / f_{2}$, the first level in fig. 5 is given by the two primary adjacents, $f_{2} / 7 \approx 514.3 \mathrm{~Hz}$ and $f_{2} / 4=$ $525 \mathrm{~Hz}$. These are subharmonics of the external frequencies and define the value of $\Delta$ in (17), i.e., $\Delta=\left(7 f_{1}-4 f_{2}\right)=$ $300 \mathrm{~Hz}$. The mediant between these two solutions corresponds to $\tilde{f}_{s}=\left(f_{1}+f_{2}\right) /(4+7) \approx 518.2 \mathrm{~Hz}$. Clearly, this is the largest stability region in this parameter interval. Observe that $\tilde{f}_{s}$ is a generalized adjacent of both $f_{1} / 4$ and $f_{2} / 7$, (equal $\Delta$ in (17)) and thus the mediant operation (18) can be implemented to obtain the second level of the generalized Farey tree i.e., $\tilde{f}_{s}+$ $f_{1} / 4=\left(2 f_{1}+f_{2}\right) /(2 \cdot 4+7)$ and $\tilde{f}_{s}+f_{2} / 7=\left(f_{1}+2 f_{2}\right) /(4+2$. $7)$. It is clear that the procedure can be iteratively applied to give successive levels of the tree. Up to the limit of the resolution of fig. 5, all resonances found are represented in some level of the generalized tree and the mediant hypothesis of hierarchical ordering is confirmed.

We have repeated the experiment with different frequency ratios; the qualitative features of the responses of the device are insensitive to this change, and plots such as fig. 5 are qualitatively the same. Moreover, they are also analogous for different input waveforms, whether of sine, square, or sawtooth waves, and do not show a qualitative dependence on the details of the circuit. These facts lead us to suggest that the behavior described is robust and universal for a whole class of systems.

\section{CONCLUSIONS}

We have investigated universal aspects of the nonlinear dynamics of three-frequency systems. We have concentrated on the three-frequency resonances that we find in such systems, and we have formulated a hypothesis about the local scaling laws for the relative sizes of different resonances: that the daughter resonance formed by the mediant is the largest in the interval between its two parents when they are generalized adjacents.

We have constructed an experimental model of a nonlinear dynamical system with three frequencies. With this circuit, made up of quasiperiodically forced coupled phase-locked loops, we have investigated the fine structure of three-frequency resonances in the dynamics. We have confirmed experimentally that for weak coupling the most important three-frequency resonance - being that with the largest plateau in the space of parameters - in the interval between two adjacent parent resonances is given by the mediant. We emphasize that since we have been interested in universal behavior, we expect that the results we have obtained are not dependent on details of the construction of the circuit, but rather represent the behavior of any dynamical system of this class.

What importance might these findings have for phase-lockedloop circuits? The advantage of finding the widest plateaux is evident: these represent the responses that are most stable to arbitrary perturbations, and thus are those that are most likely to be found in a real system. Also, it is important to understand what happens when another frequency is added in parasitic fashion to a periodically-forced system; that is one considers one of the two forces as a perturbation. This can produce undesired effects in the normal phase locking of the system, and as such from an engineering viewpoint it is important to know the regions of parameter space where the behaviour is dependable. It may be that a technique based on the simultaneous synchronization of two reference signals offers some advantages in certain technological applications. For example, it may be less prone to the influence of perturbations, or may be able to maintain the system in a certain frequency range when one of the two frequencies is 


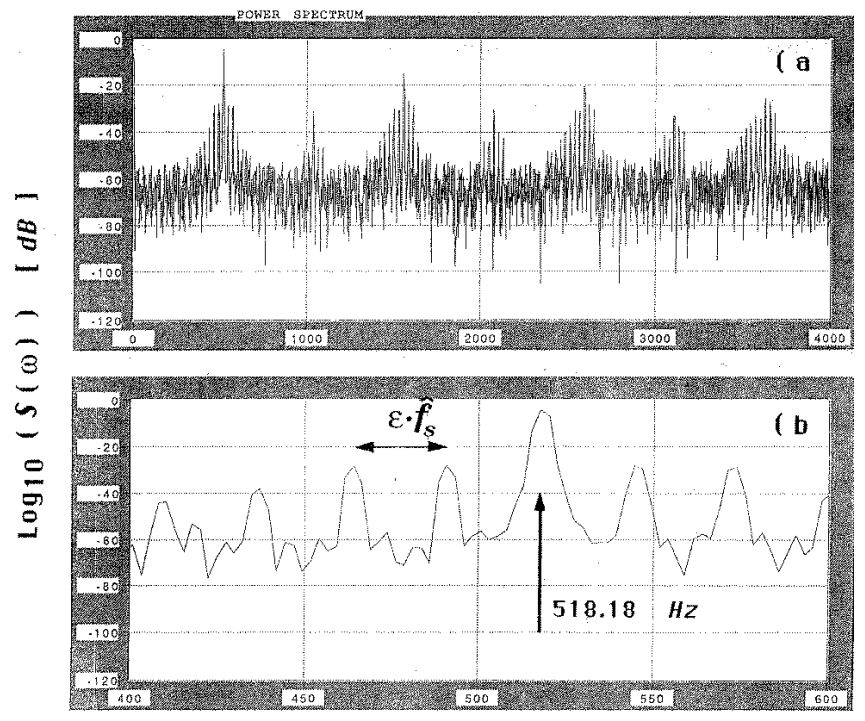

frequency $\omega[\mathrm{Hz}]$

Fig. 6. The power spectrum of the output of the cicuit for a DC offset of 0.95 V. Other parameters are as in fig. 5. (a) From $0-4000 \mathrm{~Hz}$. (b) Detail from $400-600 \mathrm{~Hz}$, showing peak at $\tilde{f}_{s} \approx 518.18 \mathrm{~Hz}$ and minor peaks separated a distance $\varepsilon \tilde{f}_{s}$.

not present, perhaps through system failure, or owing to external conditions (for example, weather conditions in the case of atmospheric propagation of radio signals). Clearly, when there is only one reference frequency, the absence of that frequency will leave the system in an unpredictable state. The presence of two reference frequencies in synchronization applications can then lead to systems that are more robust and reliable in the face of perturbations and failures.

\section{ACKNOWLEDGMENTS}

JHEC and OP acknowledge the financial support of the Spanish Dirección General de Investigación Científica y Técnica, contracts PB94-1167 and PB94-1172.

\section{REFERENCES}

[1] T. Endo and L. O. Chua, "Chaos from phase-locked loops," IEEE Trans. Circuits and Systems, vol. 35, pp. 987-1003, 1988.

[2] T. Endo and L. O. Chua, "Chaos in mutually coupled phase-locked loops," IEEE Trans. Circuits and Systems, vol. 37, pp. 1183-1187, 1990.

[3] T. Endo and L. O. Chua, "Synchronization of chaos in phase-locked loops," IEEE Trans. Circuits and Systems, vol. 38, pp. 1580-1588, 1991.

[4] T. Endo and L. O. Chua, "Piecewise-linear analysis of high-damping chaotic phase-locked loops using Melnikov's method," IEEE Trans. Circuits and Systems, vol. 40, pp. 801-807, 1993.

[5] E. Bradley, "Using chaos to broaden the capture range of a phase-locked loop," IEEE Trans. Circuits and Systems, vol. 40, pp. 808-818, 1993.

[6] E. Bradley and D. E. Straub, "Using chaos to broaden the capture range of a phase-locked loop: Experimental verification," IEEE Trans. Circuits and Systems, vol. 43, pp. 914-922, 1996.

[7] H.-A. Tanaka, S. Oishi, and K. Horiuchi, "Geometric structure of mutually coupled phase-locked loops," IEEE Trans. Circuits and Systems, vol. 43 , pp. $438-443,1996$.

[8] D. L. González and O. Piro, "Chaos in a nonlinear driven oscillator with exact solution," Phys. Rev. Lett., vol. 50, pp. 870-872, 1983.

[9] D. G. Aronson, M. A. Chory, G. R. Hall, and R. P. McGehee, "Bifurcations from an invariant circle for two-parameter families of maps of the plane: A computer-assisted study," Commun. Math. Phys., vol. 83, pp. 303-354, 1983.
[10] P. Cvitanović, B. Shraiman, and B. Söderberg, "Scaling laws for mode lockings in circle maps," Physica Scripta, vol. 32, pp. 263-270, 1985.

[11] B.-L. Hao, Elementary Symbolic Dynamics and Chaos in Dissipative Systems, World Scientific, 1989.

[12] D. K. Arrowsmith, J. H. E. Cartwright, A. N. Lansbury, and C. M. Place, "The Bogdanov map: Bifurcations, mode locking, and chaos in a dissipative system," Int. J. Bifurcation and Chaos, vol. 3, pp. 803-842, 1993.

[13] C. Baesens, J. Guckenheimer, S. Kim, and R. S. MacKay, "Three coupled oscillators: Mode-locking, global bifurcations and toroidal chaos," Physica D, vol. 49, pp. 387-475, 1991.

[14] R. van Buskirk and C. Jeffries, "Observation of chaotic dynamics of coupled nonlinear oscillators," Phys. Rev. A, vol. 31, pp. 3332-3357, 1985.

[15] A. W. Cumming and P. S. Linsay, "Quasiperiodicity and chaos in a system with three competing frequencies," Phys. Rev. Lett., vol. 60, pp. 2719$2722,1988$.

[16] P. S. Linsay and A. W. Cumming, "Three-frequency quasiperiodicity, phase locking, and the onset of chaos," Physica D, vol. 40, pp. 196-217, 1989.

[17] C. Huygens, "Extrait d'une lettre escrite de La Haye, le 26 fevrier 1665," J. des Scavans, , no. 11 (16 March), 1665, See the correction published in the following issue [18].

[18] C. Huygens, "Observation a faire sur le dernier article de precedent journal, où il est parlé de la concordance de deux pendules suspenduës à trois ou quatre pieds l'une de l'autre," J. des Scavans, , no. 12 (23 March), 1665, Huygens' notebook is reprinted in [19].

[19] C. Huygens, Euvres Complètes de Christiaan Huygens, vol. 17, p. 185, Societé Hollandaise des Sciences, 1888-1950.

[20] B. van der Pol, "Forced oscillations in a circuit with non-linear resistance (reception with reactive triode)," Phil. Mag. (7), vol. 3, pp. 65-80, 1927.

[21] B. van der Pol and J. van der Mark, "Frequency demultiplication," Nature, vol. 120, pp. 363-364, 1927.

[22] J. H. E. Cartwright, D. L. González, and O. Piro, "Universality in threefrequency resonances," Phys. Rev. E, vol. 59, pp. 2902-2906, 1999.

[23] O. Calvo, J. H. E. Cartwright, D. L. González, O. Piro, and O. Rosso, "Three-frequency resonances in dynamical systems," Int. J. Bifurcation \& Chaos, vol. 9, pp. ?-?, 1999.

[24] A. Y. Kinchin, Continued Fractions, University of Chicago Press, 1964.

[25] G. H. Hardy and E. M. Wright, An Introduction to the Theory of Numbers, Oxford University Press, fourth edition, 1975.

[26] S. Kim and S. Ostlund, "Renormalization of mappings of the two-torus," Phys. Rev. Lett., vol. 55, pp. 1165-1168, 1985.

[27] S. Kim and S. Ostlund, "Simultaneous rational approximations in the study of dynamical systems," Phys. Rev. A, vol. 34, pp. 3426-3434, 1986.

[28] G.-C. Hsieh and J. C. Hung, "Phase-locked loop techniques — a survey," IEEE Trans. Industrial Electronics, vol. 43, pp. 609-615, 1996.

[29] N. Margaris and P. Mastorocostas, "On the nonlinear behavior of the analog phase-locked loop: Synchronization," IEEE Trans. Industrial Electronics, vol. 43, pp. 621-629, 1996.

[30] K. Dessouky and W. C. Lindsey, "Phase and frequency transfer between mutually synchronized oscillators," IEEE Trans. Commun., vol. 32, pp. 110-117, 1984.

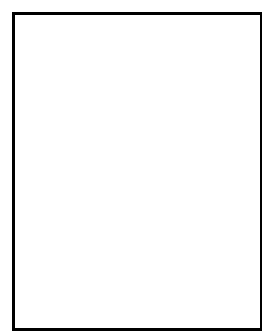

Oscar Calvo received his BSEE in electrical engineering from the National University of La Plata (Argentina) in 1979, and his MSEE in computer and electrical engineering from the Illinois Institute of Technology, (Chicago, IL, USA) in 1988. He has been a guest scientist at the Fermi National Accelerator Laboratory (Chicago, IL, USA), sponsored research staff at the Massachusetts Institute of Technology (Boston, MA, USA), and invited professor at the University of the Balearic Islands (Palma de Mallorca, Spain). He holds a position as adjunct professor and researcher at the National University of La Plata. His research is in the areas of control systems and VLSI devices. 
science.

Julyan Cartwright received his BSc in theoretical physics from the University of Newcastle upon Tyne (UK) in 1988, and his PhD in applied mathematics from Queen Mary and Westfield College of the University of London (UK) in 1992. Since then he has been working in Spain, until recently with the University of the Balearic Islands (Palma de Mallorca, Spain). He is now with the Andalusian Institute for Earth Sciences (IACT, CSIC-UGR) in Granada, Spain. His research encompasses a broad range of interdisciplinary themes within the field of nonlinear

Diego González received his BSc in physics from the National University of La Plata (Argentina) in 1981 and his $\mathrm{PhD}$ in theoretical physics from the same university in 1987. Since then he has been with the Lamel Institute (Bologna, Italy) of the Italian National Research Council (CNR). His research is mainly in the field of low-dimensional dynamical systems and its application to the modeling of complex biological systems.

Oreste Piro obtained his $\mathrm{PhD}$ in physics from the $\mathrm{Na}-$ tional University of La Plata (Argentina) in 1984. At the postdoctoral level he worked at the James Franck Institute of the University of Chicago (IL, USA), as a visiting scholar, at the Brookhaven National Laboratory (Upton, NY, USA), as a guest scientist, and at Queen Mary and Westfield College of the University of London (UK) as a senior researcher. He has been a faculty member of the National University of La Plata and fellow of the the National Council for Scientific and Technical Investigations (CONICET) in Argentina, fellow of the National Center for Scientific Research (CNRS) at the Nonlinear Institute (INLN) in Nice, France, and is currently professor at the University of the Balearic Islands and member of the Mediterranean Institute for Advanced Studies (IMEDEA, CSIC-UIB) in Mallorca, Spain. His research interests range over many areas of the theory of nonlinear dynamical systems and its applications to engineering, biology, and physics.

Francesco Sportolari received his BSEE in electronic engineering from Bologna University (Italy) in 1986. He has worked in different private companies in the area of software development. Since 1992 he has had a research contract from the Lamel Institute of the CNR (Bologna, Italy) for the modeling and visualization of complex systems. His research is mainly in the field of nonlinear modeling of complex systems with particular emphasis on simulation by means of analog electronic circuits. 